\title{
Two novel ECHS1 variants, affecting splicing and reducing enzyme activity, is associated with mitochondrial encephalopathy in infant: a case report
}

Miaojuan $\mathrm{Wu}^{1 \dagger}$, Wenqi Gao ${ }^{2 \dagger}$, Zhifang Deng ${ }^{3 \dagger}$, Zhisheng Liư ${ }^{4}$, Jiehui Ma ${ }^{4}$, Han Xiao ${ }^{2}, Y_{u} \mathrm{Xu}^{5}$ and Dan Sun ${ }^{4 *}$

\begin{abstract}
Background: Short-chain enoyl-CoA hydratase (ECHS1) is a multifunctional mitochondrial matrix enzyme involved in the second step of mitochondrial fatty acid $\beta$-oxidation. Mitochondrial diseases resulting from ECHS1 mutations are often characterised by encephalopathy, deafness, epilepsy, optic atrophy, cardiomyopathy, dystonia, and lactic acidosis. In this study, we report two novel heterogeneous variants, c.414 + 5G > A (in intron 3) and c.310C > G (in CDS), of ECHS1 in an infant with mitochondrial encephalopathy.

Case presentation: The two novel variants, C.414 + 5G > A (Chr10:135183403) in intron 3 and c.310C > G (Chr10: 135183512) in CDS, were identified by next generation sequencing (NGS). A minigene assay was used to analyse the function of the c.414 + 5G > A variant. ECHS1 enzyme activity was measured by spectrophotometry in the patient-derived myoblasts. The 2-year old patient presented with mitochondrial encephalopathy since birth. Clinical features were encephalopathy, epilepsy, and hindered psychomotor and language development. Serum lactate and blood ammonia levels were elevated, and brain magnetic resonance imaging showed abnormal signals in the bilateral frontal, parietal, and occipital cortices and brainstem and basal ganglia. We found two novel heterogeneous variants in ECHS1 in this patient. Minigene assay revealed the c.414 +5G > A variant as the cause of intronic cryptic splice site activation and 39 bp deletion in mature mRNA. In silico analysis predicted that c.310C > G might change glutamine (Q) to glutamic acid (E) in the 104th amino acid sequence (p.Q104E). To investigate the impact of these two variants on protein function, we constructed a 3D model of human ECHS1 and showed that the variants might alter the highly conserved region in close proximity to the active site, which might hinder, or even halt, enzymatic activity. The experimental assay showed that ECHS1 enzyme activity in the patient-derived myoblasts decreased compared to that in control.

(Continued on next page)
\end{abstract}

\footnotetext{
*Correspondence: bloveriver@163.com

${ }^{\dagger}$ Miaojuan Wu, Wenqi Gao and Zhifang Deng contributed equally to this work.

${ }^{4}$ Department of Pediatric Neurology, Wuhan Children's Hospital (Wuhan Maternal and Child Healthcare Hospital), Tongji Medical College, Huazhong University\&Technology, Wuhan, Hubei, China

Full list of author information is available at the end of the article
}

(c) The Author(s). 2020 Open Access This article is licensed under a Creative Commons Attribution 4.0 International License, which permits use, sharing, adaptation, distribution and reproduction in any medium or format, as long as you give appropriate credit to the original author(s) and the source, provide a link to the Creative Commons licence, and indicate if changes were made. The images or other third party material in this article are included in the article's Creative Commons licence, unless indicated otherwise in a credit line to the material. If material is not included in the article's Creative Commons licence and your intended use is not permitted by statutory regulation or exceeds the permitted use, you will need to obtain permission directly from the copyright holder. To view a copy of this licence, visit http://creativecommons.org/licenses/by/4.0/. The Creative Commons Public Domain Dedication waiver (http://creativecommons.org/publicdomain/zero/1.0/) applies to the data made available in this article, unless otherwise stated in a credit line to the data. 
(Continued from previous page)

Conclusions: Our findings are the first to report a mitochondrial encephalopathy infant carrying two novel ECHS1 variants, c.414 + 5G > A and c.310C > G, which might be deleterious variants, function as pathogenicity markers for mitochondrial encephalopathy, and facilitate disease diagnosis.

Keywords: Mitochondrial encephalopathy, ECHS1, Variants

\section{Background}

Mitochondrial disorders, mostly genetically heterogeneous, include different clinical phenotypes. Brain involvement is commonly found in most cases, but rarely is the unique clinical manifestation. Therefore, it is difficult to make a definitive diagnosis of mitochondrial disorders in patients, especially infants. Various genetic defects in nuclear genes that encode mitochondrial proteins could cause mitochondrial dysfunction, ultimately resulting in mitochondrial diseases. It is important for medical staff to know the association between clinical manifestations and genetic testing of mitochondrial disorders to establish accurate diagnoses.

$E C H S 1$, a nuclear gene, is located on chromosome 10q26.2-q26.3 [1, 2]. The ECHS1 gene encodes the mitochondrial short-chain acryloxyethyl CoA hydratase (short-chain enoyl-CoA hydratase, SCEH, or ECHS1), which is localised in the mitochondrial matrix and catalyses the hydration of enoyl-CoA in many metabolic pathways, including short-chain fatty acid $\beta$-oxidation, branched-chain amino acid catabolism, and mitochondrial enzyme catalytic unsaturated fatty acids [3, 4]. ECHS1 has been extensively investigated in model organisms due to its special role in mitochondrial oxidation; however, the relationship between ECHS1 and infant health and disease is obscure.

In this study, we reported two novel ECHS1 variants and provided experimental evidence that attests to the functional significance of these variants and their association with severe encephalopathy.

\section{Case presentation}

A 2-year old patient was hospitalised in Wuhan Children's Hospital (Wuhan Maternal and Child Healthcare Hospital). Signatures of written informed consent forms by the patients and their family members were acquired, and the study was approved by the review boards of the ethics institutions.

The patient reported was an infant boy with healthy and non-related parents, born after a 40-week pregnancy (weight $3500 \mathrm{~g}$, length $51 \mathrm{~cm}$ ). This patient was the first affected child in his family (his parents had two children, his elder brother was unaffected). His parents were both Chinese and not cousins. When he was born, a hug-like disease attack began, occurring 5-6 times a day, frequently at night. Subsequently, at 1 month of age, the patient was hospitalised due to neonatal pneumonia, neonatal jaundice, and left hydronephrosis. Psychomotor developmental delay was noted at 8 months of age. He could not raise his head at 8 months of age, and could not sit alone at 13 months of age, or speak a meaningful word when he was 2 years old, with muscle hypotonia and spasticity becoming prominent after the first year.

Electromyography (EMG) and repeated EEG recordings were normal. Laboratory tests showed elevated lactic acid- $11.41 \mathrm{mmol} / \mathrm{L}$ (normal range $0.5-2.22 \mathrm{mmol} / \mathrm{L}$ )and blood ammonia- $201 \mu \mathrm{mol} / \mathrm{L}$ (normal range 18$72 \mu \mathrm{mol} / \mathrm{L}$ ). Blood acylcarnitine analysis showed no abnormality. The value of blood total ketone body of this infant is $0.2 \mathrm{mmol} / \mathrm{L}$. Urinary organic acid profiling revealed elevated 3-hydroxybutyrylcarnitine excretion. Urine analysis showed $\mathrm{KET}^{3+}$, $\mathrm{BIL}^{1+}$, and $\mathrm{PRO}^{1+}$. A positive result in the fecal occult blood test suggested that intestinal mucosal damage was present. Myocardial enzyme, electrolyte, T3, T4, and TSH levels were within the normal range, and liver and renal functions were basically normal. Our diagnosis was "mitochondrial myopathy", and we administered the appropriate therapeutic cocktail for about 3 months. After the treatment, there was significant improvement in the symptoms, especially in the hug-like episode frequency.

Brain magnetic resonance imaging showed diffuse, long T1 and T2 signals distributed symmetrically in the cortex, brainstem, and basal ganglia of the double forehead occipital lobe. The lateral ventricles were slightly enlarged, and the cerebral sulcus was slightly wider. There was no obvious abnormal signal in the cerebellum of the posterior fossa and no shift in the midline structure (Fig. 1). The MRS results indicated a decrease in NAA with a Ch/NAA of 3.47 (Fig. 2).

\section{Functional analysis of c.414 + 5G > A and c.310C > G variants}

Next generation sequencing was used to analyse the whole exome of the patient with suspected mitochondrial disorders. A noncoding rare heterozygous variant c. $414+5 G$ > A (chr10: g. 135,183,403) in intron 3 and a novel heterozygous variant c.310C > G (Chr10: 135183512) in CDS of the ECHS1 [MIM \#602292] gene were found. No other changes were observed in the studied region. Because most mitochondrial diseases caused by nuclear DNA mutations are inherited in an 
A

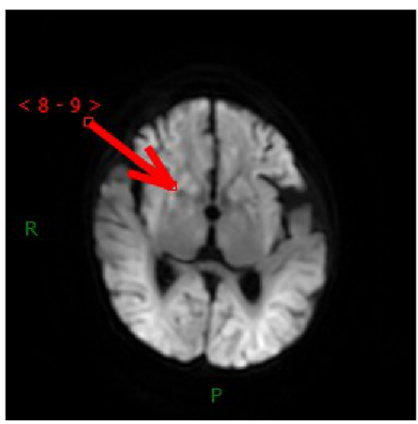

C

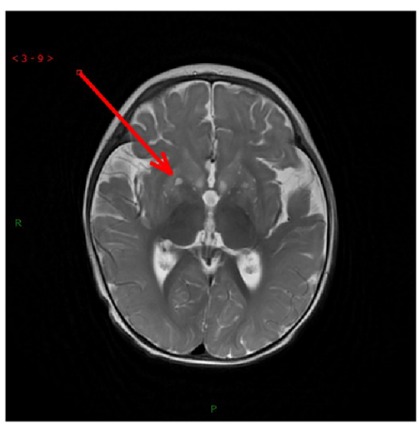

E

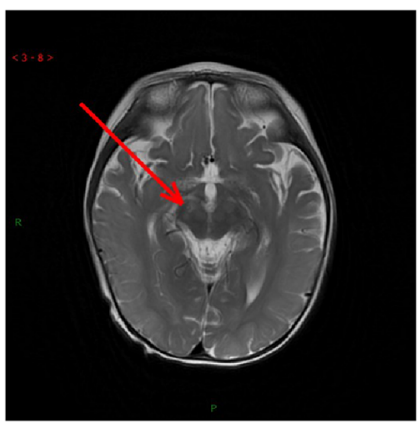

B

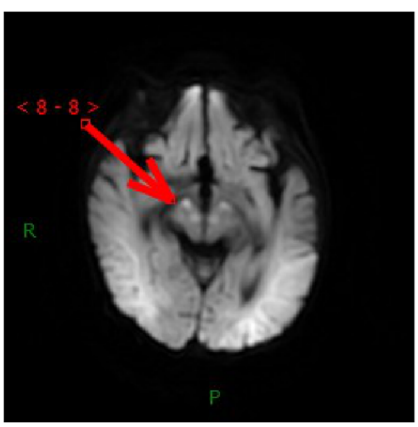

D

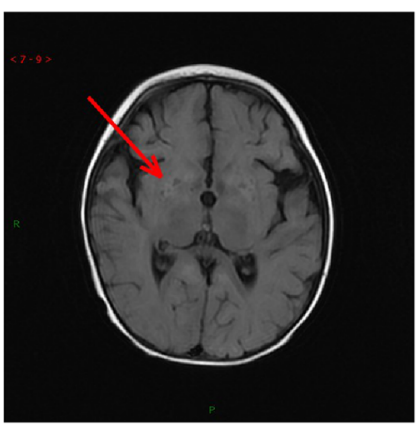

F

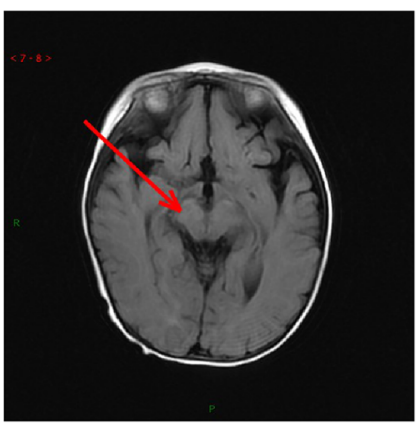

Fig. 1 The MRI assay of the patient. (a-b) DWI image; (c-d) T2 image;(e-f)T2 FLAIR image

autosomal recessive manner, the two novel variants seemed particularly peculiar. We proceeded to verify the genomic ECHS1 DNA from both the patient and his parents using Sanger sequencing. The results revealed no other ECHS1 variants. Furthermore, the patient's father was heterozygous for only one variant, c. $414+$ $5 \mathrm{G}>\mathrm{A}$, and the mother for only the other variant, c. $310 \mathrm{C}>\mathrm{G}$, indicating that the patient inherited one variant from each parent, and that both mutant alleles were expressed (Fig. 3). The information on the two novel variants is summarised in Table 1.

We predicted the variant c. $310 \mathrm{C}>\mathrm{G}$ function by Phyre2 (http://www.sbg.bio.ic.ac.uk/phyre2/html/page.cgi?id=index) and I-TASSER (https://zhanglab.ccmb.med.umich.edu/ITASSER/). The analysis indicated that this variant might change glutamine (Q) to glutamic acid (E) in the 104th amino acid sequence (p.Q104E).
Because c. $414+5 \mathrm{G}>\mathrm{A}$ was located in an intron, we first performed bioinformatic analysis using the Human Splice Finder, Splice Port, and Fruit Fly Splice Predictor. The results showed high probability for intronic cryptic splice site activation, leading to $39 \mathrm{bp}$ deletion. Splice site score calculator (http://rulai.cshl.edu/new_alt_exon_ $\mathrm{db} 2 / \mathrm{HTML} / \mathrm{score} . \mathrm{html}$ ) was used to assess the strength of the constitutive and cryptic acceptor splicing sites, yielding 14.2 and 7.9, respectively. To prove that the 39 bp deletion was caused by the c. $414+5 \mathrm{G}>\mathrm{A}$ variant, we then conducted the minigene-based splicing experiment. The minigene splicing products were analysed by PCR amplification with plasmid-specific primers and visualised with polyacrylamide gel electrophoresis (Fig. 4a). The electrophoresis results of wild-type and c.414+ $5 \mathrm{G}>\mathrm{A}$ transfections indicated that the c. $414+5 \mathrm{G}>\mathrm{A}$ variant exerted significant effects on splicing patterns. 


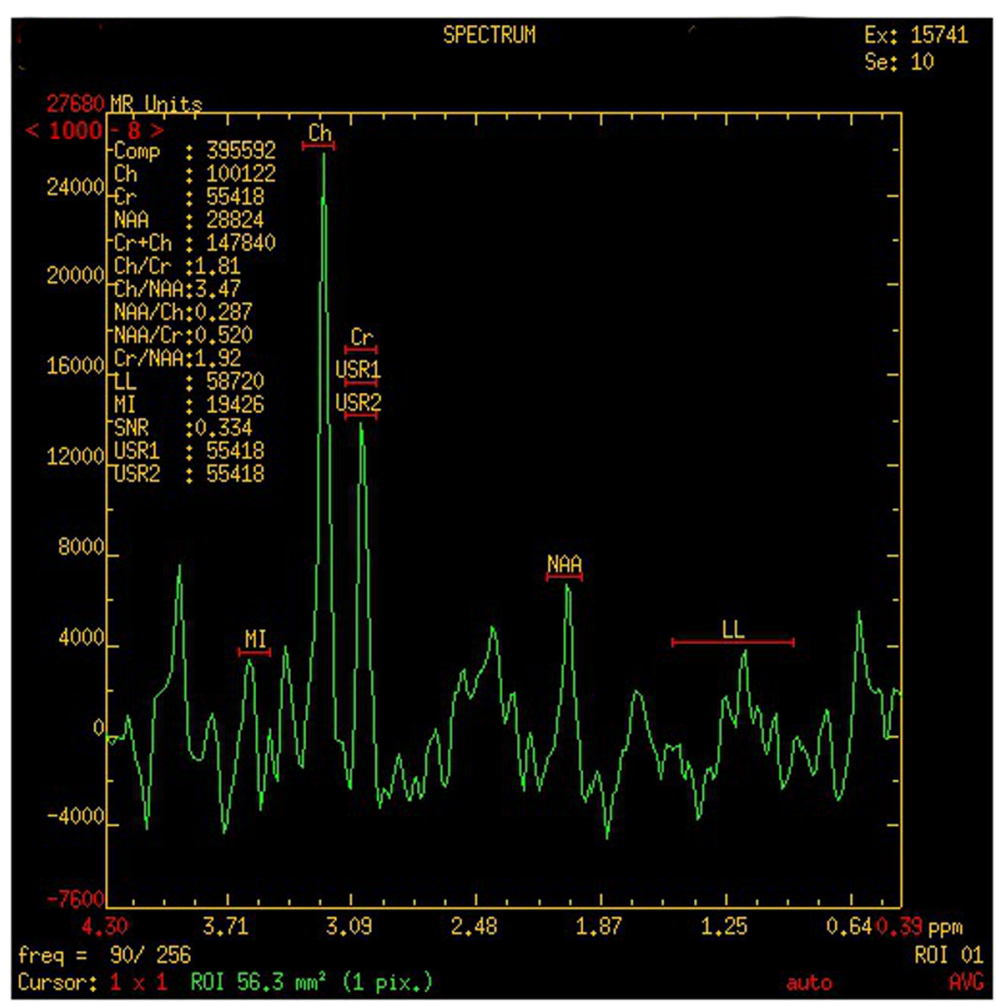

Fig. 2 The MRS assay of the patient

The amplicons confirmed by Sanger sequencing showed that the cDNA fragment obtained from the c. $414+5 \mathrm{G}>$ A plasmid contained the same $39 \mathrm{bp}$ deletion as the cDNA of the patient (Fig. 4c).

In silico analysis of c.414 + 5G $>A$ and c.310C > G variants in protein function

To predict the impact of $39 \mathrm{bp}$ deletion and p.Q104E caused by c. $414+5 \mathrm{G}>\mathrm{A}$ and c. $310 \mathrm{C}>\mathrm{G}$ on protein function, we performed in silico analysis. VariantValidator [5] was used to verify the annotation of the Chr10: 135183403 deletion. The Human Splice Finder [6], Splice Port [7], and Fruit Fly Splice Predictor [8] were used to assess the predicted effect on the splicing site. SMART analysis (http://smart.embl-heidelberg.de) suggested that the catalytic domains of the core enzyme comprised amino acids 42 to 290 in the ECHS1 sequence. The $39 \mathrm{bp}$ deletion in mature mRNA might cause a 13 amino acid-deletions (from amino acid 126 to $138)$ in the core enzyme catalytic region of ECHS1. The 13 missing amino acids and p.Q104E were both located in the protein active site. Subsequently, PyMol software was used to construct the 3D model of ECHS1 (Fig. 5). Such alteration with the involvement of highly conservative amino acids probably had a crucial impact on protein activity. Therefore, we speculated that the mutations severely reduced ECHS1 enzyme activity.

\section{Measurement of ECHS1 enzyme activity in patient's} myoblasts

Based on the above computer prediction, in order to test the exact functional impact of the novel variants, we proceeded to determine if ECHS1 enzyme activity changed in the mitochondria of patient' myoblasts. ECHS1 enzyme activity in the patient-derived primary fibroblast cell lysates was determined by spectrophotometry, followed by measuring the absorbance of the unsaturated substrate crotonyl-CoA over time $(15 \mathrm{~min})$. The experimental procedure was performed according to previous publications [9]. The experiments were performed in triplicates. Error bars represent standard deviations. Our results showed that ECHS1 enzyme activity in mitochondrial fractions of myoblast cell lysates from patients was remarkably reduced when normalised to control activity. ECHS1 enzyme activity from patients decreased to $28 \%$ of the normal value (Fig. 6). Overall, the variants might generate a serious depletion of ECHS1 enzyme activity.

\section{Discussion and conclusions}

In this report, we identified a 2-year old patient harbouring two novel heterozygous variants, c. $414+5 \mathrm{G}>\mathrm{A}$ and c. $310 \mathrm{C}>\mathrm{G}$, in ECHS1. To our knowledge, our study is the first to report the c.414 + 5G > A and c. $310 \mathrm{C}>\mathrm{G}$ variants, and provided the first experimental 

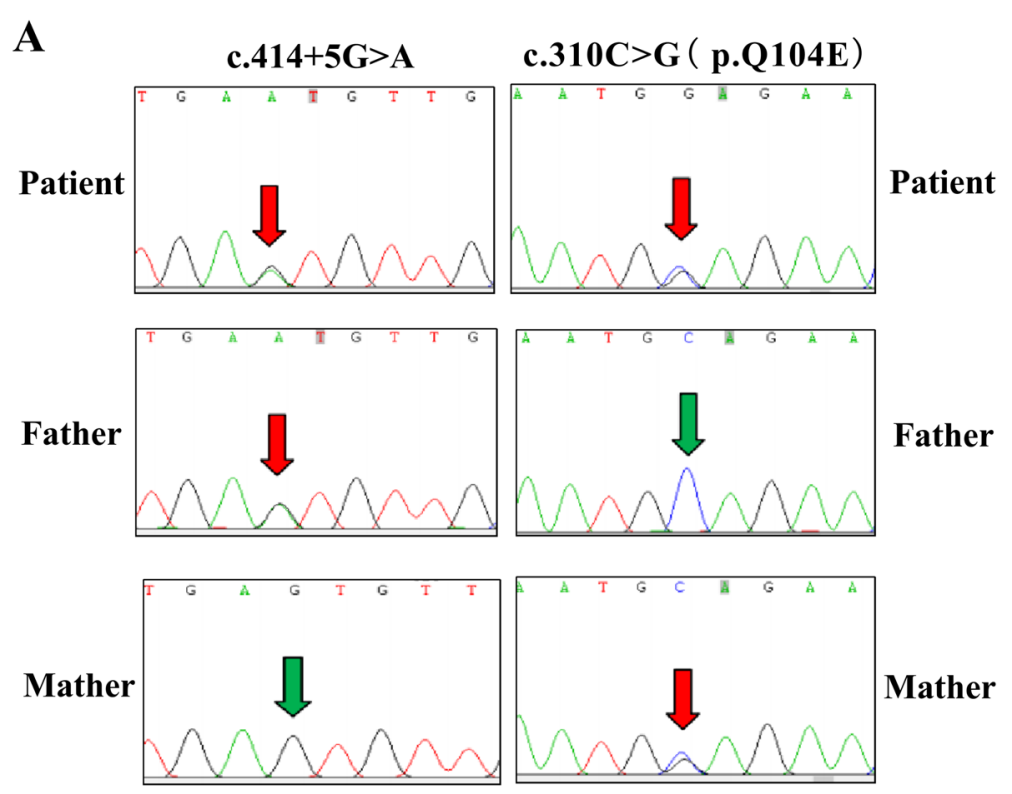

B

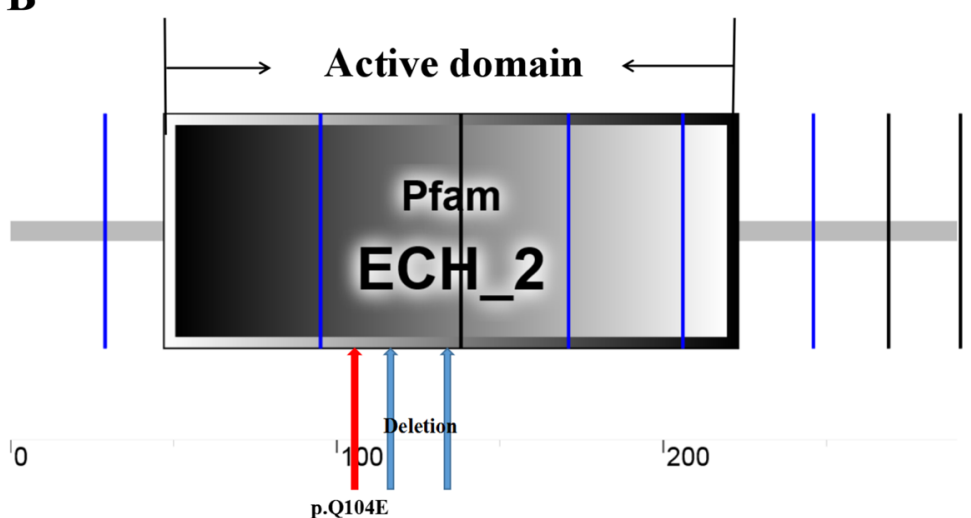

Fig. 3 ECHS1 Sanger sequence analysis and ECHS1 functional domain. a Sanger sequencing of genomic DNA of patient and his parents. $\mathbf{b} \mathrm{A}$ schematic diagram of the functional domains in ECHS1 and the locations of the mutation and deletion

characterisation of the c. $414+5 \mathrm{G}>\mathrm{A}$ variant, elucidating its impact on splicing. The c.310C $>\mathrm{G}$ variant in the CDS region might result in a single amino acid mutation, changing glutamine $(\mathrm{Q})$ into glutamic acid (E) in the number 104th amino acid sequences (p.Q104E). The c.414+5G > A variant was located in intron 3 , and the variant might bring about 13 amino acid deletions. 3D structure prediction of ECHS1 showed that p.Q104E mutation and 13 amino acid deletions might destroy the activity domain. Surprisingly, we found that ECHS1 enzyme activity was lower in patient-derived myoblasts than in the control, which corroborates the previous speculation.

ECHS1 deficiency, as reported previously, is mostly characterised by the following prominent neurological symptoms: sensorineural deafness, developmental retardation,

Table 1 The information of variants

\begin{tabular}{llllllll}
\hline Gene name & $\begin{array}{l}\text { Chromosome localization } \\
\text { (GRCh37/hg19) }\end{array}$ & Variant & NM & Location & $\begin{array}{l}\text { Homozygosis/ } \\
\text { Heterozygosis }\end{array}$ & $\begin{array}{l}\text { Amino acid } \\
\text { alteration }\end{array}$ & $\begin{array}{l}\text { Inheritance model } \\
\text { Variation } \\
\text { source }\end{array}$ \\
\hline ECHS1 & Chr10:135183403 & c.414+5G > A & NM_004092.3 & Intron3 & Heterozygosis & N/A & $\begin{array}{l}\text { Autosomal recessive Father } \\
\text { inheritance }\end{array}$ \\
& Chr10:135183512 & c.310C>G & CDS & Heterozygosis & p.Q104E & $\begin{array}{l}\text { Autosomal recessive } \\
\text { inheritance }\end{array}$ \\
\hline
\end{tabular}




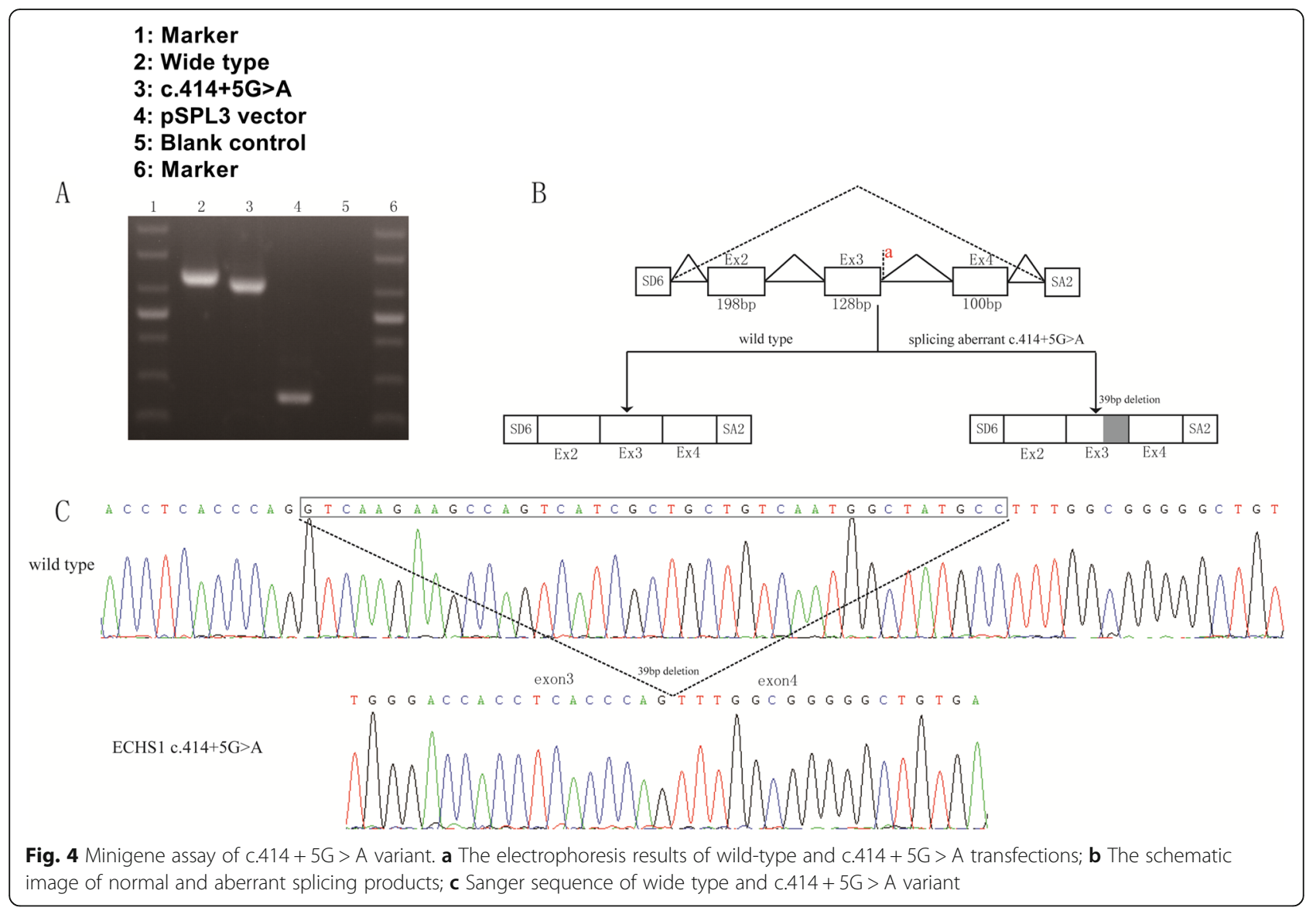

epileptic seizures, optic atrophy, and hypotonia. Other clinical features include cardiomyopathy, respiratory insufficiency, and elevated lactic acid levels in blood, urine, and cerebrospinal fluid. MRI images of the brain are similar to those of Leigh syndrome, with white matter changes, symmetrical high T2 signal in the bilateral basal ganglia, and brain atrophy $[10,11]$. In our report, the patient presented with similar symptoms that were indicative of neurologic disorders, as well as elevated lactic acid levels. Regarding the association between ECHS1 genetic variants and diseases, previous publications have reported different types of variants in patients. There were 34 pathogenic mutant forms, among which 29 were missense mutations, 3 were splicing mutations, 1 was a code shift mutation, and 1 was a nonsense mutation $[1,12]$. Missense mutations and complex heterozygous mutations were found in the majority of the cases. Homozygous mutations, mostly found in exon 4, exon 5 and exon 6 as well as in exon 8, and splicing
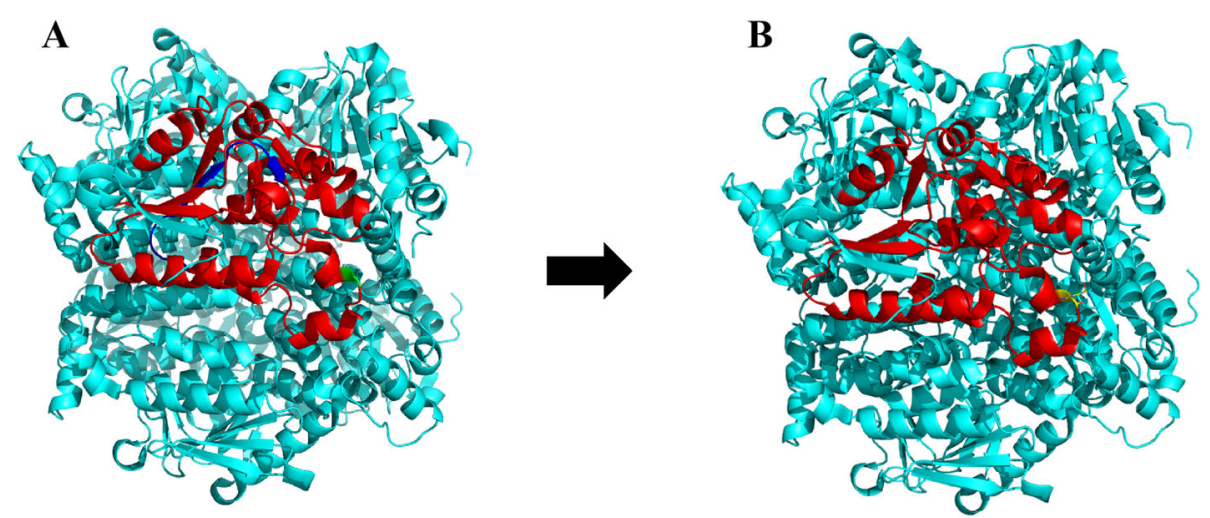

Fig. 5 3D model of the human ECHS1. a Wide type, red-confidently predicted active domain, blue-deleted amino acids, green-glutamine (Q); b Mutated type, the number 126-138 amino acids deleted, yellow-glutamic acid (E) 


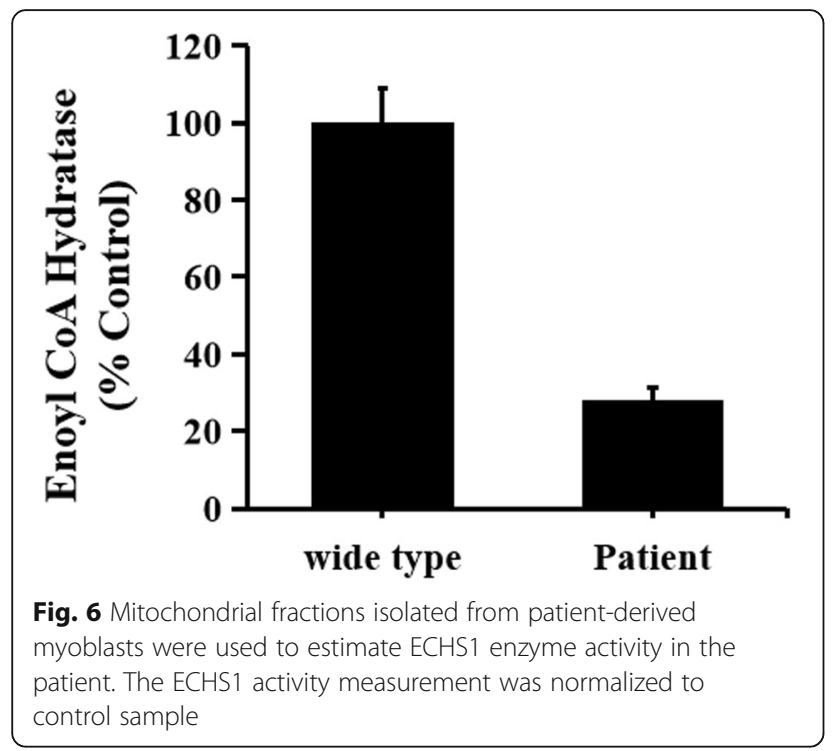

mutations, were also found in some cases. The most frequently studied variants were c.476A $>$ G (p.Gln159Arg), c.538A > G (p.Thr180Ala), and c.817A > G (p.Lys273Glu) $[1,3,13,14]$. Many types of variants were found; however, no clear association between the clinical phenotype and genotype of ECHS1 was found. We presently found two novel variants, c. $414+5 \mathrm{G}>\mathrm{A}$ and c.310C $>\mathrm{G}$, in the $E C H S 1$ gene, and no other variants were found in the study region. Both computer prediction and experimental analysis showed that ECHS1 enzyme activity was decreased in the patient's myoblasts. However, there were some limitations to our study. First, we lacked an in vitro experiment, especially exogenous expression of the respective mutant ECHS1 protein in cancer cells, to verify the exact effects of these two variants on ECHS1 expression and enzyme activity. Second, a rescue assay is needed. Inserting wild-type ECHS1 into immortalised patient-derived myoblasts to observe the enzyme activity level and mitochondrial function, which requires several more patients, in order to determine the detailed association between these variants and disease.

In conclusion, two novel c. $414+5 \mathrm{G}>\mathrm{A}$ and c. $310 \mathrm{C}>$ $G$ variants leading to decreased ECHS1 activity were identified in our study. Establishing a diagnosis in patients with a proposed mitochondrial disorder is often a challenge, especially in paediatric cases. Therefore, we hope that these pathogenic variants could serve as biomarkers for mitochondrial encephalopathy, making the diagnosis of mitochondrial diseases simpler, more convenient, and more accurate.

\section{Abbreviations}

ECHS1: Enoyl-CoA hydratase; MRI: Magnetic Resonance Imaging;

MRS: Magnetic Resonance Spectrum; EMG: Electromyography

\section{Authors' contributions}

MJ W and WQ G developed the experimental part of the project and performed under the guidance of ZF D and DS. ZS L, JH M, and HX performed in silicon analysis under the guidance of ZF D and DS. YX performed the language correction. DS analyzed the data and wrote the paper. All authors read and approved the manuscript.

\section{Funding}

The National Key Research and Development Program of China (2016YFC1306202); Wuhan Science and Technology Innovation PlatformChildren Nerve Disease Clinical Medical Research Center Funded Projects (2014-160); .

A Multicenter Clinical Study of Ketogenic Diet in the Treatment of Mitochondrial Epilepsy (FTCSF-2018-05);

The role of the funding body: Genetic analysis, minigene assay, and ECHS1 enzyme activity measurement were supported by the fundings.

\section{Availability of data and materials}

We declared that materials described in the manuscript, including all relevant raw data, will be freely available to any scientist wishing to use them for non-commercial purposes, without breaching participant confidentiality.

\section{Ethics approval and consent to participate}

Our present study protocol was approved by the Institutional Review Boards of the Wuhan Children's Hospital and performed in accordance with the Declaration of Helsinki. The family members provided written consent for themselves to participate in genetic analysis. The patient and his parents all agreed to publish this study via written consent. The consent was obtained from a parent of legal guardian of the patient. The consent was obtained from any other family members whose clinical details were discussed in this manuscript, in this section.

\section{Consent for publication}

The family members provided written consent for themselves to participate in genetic analysis. The patient and his parents all agreed to publish this study via written consent. The consent was obtained from a parent of legal guardian of the patient. The consent was obtained from any other family members whose clinical details were discussed in this manuscript, in this section.

\section{Competing interests}

The authors declare that they have no competing interests.

\section{Author details}

${ }^{1}$ Jianghan University, Wuhan, Hubei, China. ${ }^{2}$ Institute of Maternal and Child Health, Wuhan Children's Hospital (Wuhan Maternal and Child Healthcare Hospital), Tongji Medical College, Huazhong University\&Technology, Wuhan, Hubei, China. ${ }^{3}$ Department of Pharmacy, The Central Hospital of Wuhan, Tongji Medical College, Huazhong University of Science\&Technology, Wuhan, Hubei, China. ${ }^{4}$ Department of Pediatric Neurology, Wuhan Children's Hospital (Wuhan Maternal and Child Healthcare Hospital), Tongji Medical College, Huazhong University\&Technology, Wuhan, Hubei, China. ${ }^{5}$ Department of Nosocomial Infection, Wuhan Children's Hospital, Tongji Medical College, Huazhong University\&Technology, Wuhan, Hubei, China.

Received: 6 November 2019 Accepted: 20 April 2020

Published online: 30 April 2020

\section{References}

1. Sharpe AJ, McKenzie M. Mitochondrial fatty acid oxidation disorders associated with short-chain Enoyl-CoA Hydratase (ECHS1) deficiency. Cells. 2018;7(6).

2. Janssen U, Davis EM, Le Beau MM, Stoffel W. Human mitochondrial enoylCoA hydratase gene (ECHS1):structural organization and assignment to chromosome 10q26. 2-q26. 3. Genomics. 1997:40(3):470-5.

3. Al Mutairi F, Shamseldin HE, Alfadhel M, Rodenburg RJ, Alkuraya FS. A lethal neonatal phenotype of mitochondrial short-chain enoyl-CoA hydratase-1 deficiency. Clin Genet. 2017;91(4):629-33.

4. Yamada K, Naiki M, Hoshino S, Kitaura Y, Kondo Y, Nomura N, Kimura R, Fukushi D, Yamada Y, Shimozawa N, Yamaguchi S, Shimomura Y, Miura K, 
Wakamatsu N. Clinical and biochemical characterization of 3hydroxyisobutyryl-CoA hydrolase (HIBCH) deficiency that causes Leigh-like disease and ketoacidosis. Mol Genet Metab Rep. 2014;1:455-60.

5. Freeman PJ, Hart RK, Gretton LJ, Brookes AJ, Dalgleish R. Variant validator: accurate validation, mapping, and formatting of sequence variation descriptions. Hum Mutat. 2018;39(1):61-8.

6. Desmet FO, Hamroun D, Lalande M, Collod-Beroud G, Claustres M, Beroud C. Human splicing finder: an online bioinformatics tool to predict splicing signals. Nucleic Acids Res. 2009;37(9):e67.

7. Dogan RI, Getoor L, Wilbur WJ, Mount SM. Splice Port-an interactive splice-site analysis tool. Nucleic Acids Res. 2007;35(Web Server issue):W28591.

8. The Berkeley Drosophila Genome Project (BDGP) is a consortium of the Drosophila Genome Center funded by the National Human Genome Research Institute and the National Institute of General Medical Sciences through its support of work in the Susan Celniker, J. Ben Brown, Erwin Frise and Gary Karpen laboratories. http://www.fruitfly.org/.

9. Fong JC, Schulz H. Purification and properties of pig heart crotonase and the presence of short chain and long chain enoyl coenzyme a hydratases in pig and Guinea pig tissues. J Biol Chem. 1977;252(2):542-7.

10. Luís PB, Ruiter JP, Ofman R, ljist L, Moedas M, Diogo L, Garcia P, de Almeida IT, Duran M, Wanders RJ, Silva MF. Valproic acid utilizes the isoleucine breakdown pathway for its complete beta-oxidation. Biochem Pharmacol. 2011;82(11):1740-6.

11. Yamada K, Aiba K, Kitaura Y, Kondo Y, Nomura N, Nakamura Y, Fukushi D, Murayama K, Shimomura Y, Pitt J, Yamaguchi S, Yokochi K, Wakamatsu N. Clinical, biochemical and metabolic characterisation of a mild form of human short-chain enoyl-CoA hydratase deficiency: significance of increased N-acetyl-S-(2-carboxypropyl) cysteine excretion. J Med Genet. 2015:52(10):691-8.

12. Carlston CM, Ferdinandusse S, Hobert JA, Mao R, Longo N. Extrapolation of variant phase in mitochondrial short-chain Enoyl-CoA Hydratase (ECHS1) deficiency. JIMD Rep. 2019;43:103-9.

13. Haack TB, Jackson CB, Murayama K, Kremer LS, Schaller A, Kotzaeridou U, de Vries MC, Schottmann G, Santra S, Büchner B, Wieland T, Graf E, Freisinger P, Eggimann S, Ohtake A, Okazaki Y, Kohda M, Kishita Y, Tokuzawa Y, Sauer S, Memari Y, Kolb-Kokocinski A, Durbin R, Hasselmann O, Cremer K, Albrecht B, Wieczorek D, Engels H, Hahn D, Zink AM, Alston CL, Taylor RW, Rodenburg RJ, Trollmann R, Sperl W, Strom TM, Hoffmann GF, Mayr JA, Meitinger T, Bolognini R, Schuelke M, Nuoffer JM, Kölker S, Prokisch H, Klopstock T. Deficiency of ECHS1 causes mitochondrial encephalopathy with cardiac involvement. Ann Clin Transl Neurol. 2015;2(5):492-509.

14. Fitzsimons $\mathrm{PE}$, Alston $\mathrm{CL}$, Bonnen PE, Hughes J, Crushell E, Geraghty MT, Tetreault M, O'Reilly P, Twomey E, Sheikh Y, Walsh R, Waterham HR, Ferdinandusse S, Wanders RJA, Taylor RW, Pitt JJ, Mayne PD. Clinical, biochemical, and genetic features of four patients with short-chain enoylCoA hydratase (ECHS1) deficiency. Am J Med Genet A. 2018;176(5):1115-27.

\section{Publisher's Note}

Springer Nature remains neutral with regard to jurisdictional claims in published maps and institutional affiliations.

Ready to submit your research? Choose BMC and benefit from:
- fast, convenient online submission
- thorough peer review by experienced researchers in your field
- rapid publication on acceptance
- support for research data, including large and complex data types
- gold Open Access which fosters wider collaboration and increased citations
- maximum visibility for your research: over 100M website views per year
At BMC, research is always in progress.
Learn more biomedcentral.com/submissions

